\title{
LA TELEVISIÓN UNIVERSITARIA POR INTERNET: ANÁLISIS DE LA SITUACIÓN ESPAÑOLA
}

\section{UNIVERSITY TV THROUGH INTERNET: ANALYSIS OF THE SPANISH SITUATION}

\author{
Julio Cabero-Almenara; cabero@us.es \\ Laura García Pereira; lauragarciapereira2@gmail.com \\ Pilar Query del Moral; querypili93@gmail.com \\ Diego García de la Concha; dgarciadelaconcha@us.es \\ Universidad de Sevilla
}

\section{RESUMEN}

Con Internet la televisión se ha transformado en diferentes direcciones: su acceso a través de distintos dispositivos, su convergencia con tecnologías de la web 2.0 y los "social media". Tal unificación ha traído la denominada TVIP o bajo streaming, en la cual las Universidades han desarrollados diferentes canales. En el artículo se analizan las TVIP de Universidades españolas, efectuándose para ello un análisis de contenido. Se concluye que el medio está adquiriendo progresiva importancia, y se presentan sus características y servicios que ofrecen. Se apuntan también algunas limitaciones de estas cadenas.

PALABRAS CLAVE: Televisión, televisión universitaria, televisión por Internet.

\section{ABSTRACT}

Internet television has transformed in different directions: access via different devices, its convergence with web 2.0 technologies and "social media". Such unification brought under the so-called IPTV or streaming, in which the universities have developed different channels. In the article the IPTV Spanish Universities are analyzed effected to do a content analysis. We conclude that the medium is gaining increasing importance, and its features and services offered are presented. Some limitations of these chains are also targeted.

KEYWORDS: Television, university television, Internet TV. 


\section{LA TELEVISIÓN COMO MEDIO DE COMUNICACIÓN}

La televisión ha sido uno de los medios de comunicación más influyentes durante el siglo XX; y ha sido una de las tecnologías más alabadas, criticadas y analizadas en el universo mediático contemporáneo. Tal ha sido su influencia que el aparato configuraba, mediante su ubicación, el espacio doméstico, y en consecuencia las relaciones sociales que se establecían en la familia.

La aparición de Internet, y diversos medios asociados a ella, como los "social media" y la web 2.0, han tenido una fuerte repercusión para transformar el escenario mediático, y modificar las audiencias, las maneras en las cuales nos acercamos a las tecnologías, y el papel que desempeñamos con las mismas.

Para Cabero $(2015,15)$ : "en esta transformación la televisión no es un medio a olvidar. La historia de la comunicación ha puesto claros ejemplos de que la aparición de una nueva tecnología de la información no nos lleva a un desplazamiento completo de la precedente, salvo en el caso de que se trate de una tecnología de sustitución. Así la radio, no mató a la prensa, el cine no mató al teatro, y la televisión ni mató en su momento al cine ni a la radio. Lo que sí ocurre es que se da una reubicación de las funciones que desempeñan los medios y los dispositivos con que se cuentan para cubrir esas funciones". Y de nuevo la historia se repite con Internet, que no ha hecho desaparecer la televisión, sino que la ha reubicado en el escenario mediático. Valga como ejemplo de lo que decimos el trabajo de Brubaker (2010), que pone de manifiesto como a la hora de acceder a las informaciones políticas, tendemos a combinar ambos medios.

Hace ya tiempo Cabero (2005) apuntaba que estaban apareciendo nuevos elementos, que iban a repercutir en la concreción y reubicación del medio televisivo en el escenario comunicativo: la aparición de nuevas tecnologías en el universo mediático, el surgir de nuevos y diferentes tipos de usuarios, y de nuevas modalidades de utilización de las TIC. Diez años después, la transformación ha sido más profunda de lo que se pensaba, produciéndose cambios de tal calado, debidos a "las nuevas prácticas de acceso a los contenidos audiovisuales que observamos sobre todo entre las jóvenes generaciones. Todos los estudios realizados sobre las nuevas prácticas de uso de la televisión en Estados Unidos y en Europa indican un cambio acelerado. Los jóvenes televidentes pasan del consumo "lineal" de TV hacia un consumo en "diferido" y "a la carta" en una "segunda pantalla"(ordenador, Tablet o Smartphone)." (Ramonet, 2015, 1).

Claro ejemplo de lo que hablamos son los datos aportados por la "Asociación de Investigación en Medios de Comunicación" española en el 2010, que encontraron que uno de cada tres encuestados, veía la televisión a través de Internet (González y López, 2011).

Estos datos, han variado en los últimos tiempos, debido a la convergencia tecnológica está llevando la televisión a una verdadera galaxia transmedia, donde sus mensajes pueden observase a través de diferentes pantallas: receptores de televisión, pantallas 
de ordenadores, ordenadores portátiles, tablet, y smaphones; sin lugar a dudas nos estamos moviendo hacia la sociedad de las "cuatro pantallas" (Artopolus, 2011; Aguaded, Sánchez y Marín, 2012). Como llama la atención Scolari (2014, 8): "Seguimos siendo grandes consumidores de contenidos audiovisuales, pero los vemos en una amplia variedad de dispositivos y pantallas. La televisión tradicional -la del broadcasting - no desaparece, mas deja de ser la única forma de consumir contenidos audiovisuales. ... Las nuevas generaciones tienden a ver cosas diferentes, en diferentes medios y con diferentes modalidades de consumo (en vivo, streaming, descargas, etc.)."

Como apunto Torres (2014), la convergencia a la que estamos haciendo referencia está permitiendo que la televisión sea móvil y social,; ya que por un parte puede ser vista a través de diferentes dispositivos en diferentes lugares, y social, puesto que nos facilita la discusión de sus contenidos, a través de los dispositivos móviles en las redes sociales. Y ello supone, como sugería Cebrián Herreros (2004, 205): la heterogeneidad de canales integrados en ofertas de paquetes, la simultaneidad-secuencialidad convergente con canales temáticos de internet, la capacidad de interacción de canales, la interactividad y búsqueda, y la existencia de un usuario navegante y buscador exacerbado de contenidos, canales e interacciones de medios. Convergencia que implica la creación de una nueva realidad mediática, muy superior a la suma de las partes.

También han variado las formas en como interaccionamos con los programas televisivos, entre otros motivos porque hemos pasado de ser receptores pasivos, es decir de meros consumidores mediáticos, a productores-difusores de mensajes o prosumidores. Ya que cada vez las personas nos estamos convirtiendo más en emisores de mensajes, y en este caso de mensajes audiovisuales, como podemos observar en Youtube o Vimeo, donde las personas crean sus canales y ponen en ellos sus mensajes. Al mismo tiempo debemos asumir que la televisión "está dejando de ser progresivamente una herramienta de masas para convertirse en un medio de comunicación consumido individualmente, a través de diversas plataformas, de forma diferida y personalizada." (Ramonet, 2015, 1).

Mientras que la televisión tradicional "impedía el desarrollo del individuo como generador de comunicación y lo convertía en 'público' de espectáculos en los que debía encontrar los puntos de conexión para activar su atención, mientras que la telefonía -evolución lógica de la comunicación inter e intrapersonal iniciada con la Web 2.0- activa la participación directa del individuo en la construcción del entramado radial sobre la que se origina la diferencia comunicativa con respecto a otros medios." (García, Vinadel y Albuin, 86-87).

La combinación de Internet y televisión, ha favorecido la aparición de un nuevo tipo de televisión denominada como "televisión por internet", "TVIP" o "IPTV"; que podríamos considerarla como un "sistema de distribución de contenidos audiovisuales y datos a través de conexiones de banda ancha de Internet" (Said, 2009, 79). O como señalan Aguaded y Marín $(2013,121)$ : "aquella en la que el telespectador forma parte de ella, e interactúa con la programación llegando a ser parte de esta." O simplemente como 
una televisión desarrollada sobre protocolos IP, desarrollada basándose en el videostreaming.

Dicha televisión, nos ofrece además de la observación de los mensajes audiovisuales otra serie de servicios, como son: participar en las redes sociales que paralelamente se asocia a ella para analizar o valorar sus mensajes, el visionado bajo demanda, y la desubicación espacial de receptores fijos.

TVIP que constituirá el futuro de la televisión, por la extensión de la banda ancha de Internet, el aumento del número de usuarios de Internet, y la posibilidad de observarla en diferentes plataformas tecnológicas (Aguaded y Marín, 2013; Orozco, 2014; Torres, 2014; Cabero, 2015; Ramonet, 2015).

Su influencia debemos también percibirla desde los cambios radicales que incorpora en la producción de los mensajes, que vendrán marcados no tanto por el mundo televisivo tradicional, sino por las nuevas narrativas que está imponiendo Internet en el terreno audiovisual: tipología de planos específica con tendencia hacia los medios y cortos, rapidez del discurso, y con mensajes tipo píldoras informativas (Blum, 2013); o la necesidad de buscar nuevos modelos legislativos para la televisión (Cooper, 2007).

\section{LA TVIP UNIVERSITARIA}

Si las TIC, de manera general, e Internet, de forma particular, han penetrado en la educación lo han hecho inicialmente en la Universidad. Penetración que ha sido constante como se desprende de los diferentes informes "Universitic" (http://www.crue.org/Publicaciones/Paginas/Universitic.aspx?Mobile=0) realizados por la "Conferencia de Rectores de las Universidades Españolas" desde 2007.

No es nuestra pretensión centrarnos en un análisis profundo de la televisión universitaria, el lector puede encontrar información en diversos trabajos (Martínez, 1998; Aguaded et al, 2012; o Cabero, 2015); si vamos a entenderla de acuerdo con la "Asociación Brasileña de Televisión Universitaria", como aquella televisión dirigida estrictamente a la promoción de la educación, la cultura y la ciudadanía, y que puede funcionar de maneras diversas: utilizando Internet, televisión por cable, satélite, o televisión abierta. Y que presenta las siguientes características distintivas: ser una producción realizada de manera que respete las diferencias, y por tanto que sea plural, imaginativa, creativa, educadora, con una fuerte misión cultural, y con una misión hacia la alfabetización mediática. Siendo su espacio de intervención los programas didácticos, documentales, culturales, científicos e institucionales.

Por lo que se refiere a su presencia en España tenemos que señalar que se han desarrollado pocas experiencias, como la de la UNED que emite un programa semanal por la segunda cadena de televisión española, o la de la Universidad de Sevilla que emite semanalmente el programa "Universitas" por la televisión local "TVCorreo". No ocurriendo lo mismo en Iberoamérica, donde hay fuertes experiencias, como las televisiones mexicanas TV UNAM, Canal 11, y Canal 44, o el canal Zoom de Colombia. 
Frente a otros tipos de televisión, la universitaria es un modelo que debe ir dirigido hacia la innovación, la experimentación, y la creación y producción de nuevos géneros, formatos y estéticas televisivas (Cabero, 2015). Por otra parte, no debe caber la menor duda que las televisiones universitarias juegan un rol muy efectivo como espacios de innovación y experimentación, ya sea en la programación, en la formación de personal, observación, en la investigación sobre los dispositivos de en los contenidos, o en la utilización de nuevos formatos.

De todas formas no debemos olvidarnos que es una televisión institucional, que establece como principio el respeto a las diferencias y tiende a lo plural, hacia la imaginación y la creatividad, es educadora, con una fuerte misión cultural, y que debe propiciar la alfabetización mediática. Por tanto hablar de televisión universitaria, es hablar de una televisión que debe defender la cultura, la educación y la ciencia; y de una televisión que debe dar cabida a la participación de todos los sectores universitarios: docentes, discentes y personal de administración y servicio.

Señalar que la aparición de la TVIP ha repercutido para que las Universidades españolas impulsen la creación de televisiones universitarias, que han surgido desde diferentes modelos: asociad a las facultades de ciencias de la información, relacionadas con los gabinetes de prensa de las universidades, incorporadas a los servicios de informática, o asociadas a los servicios de producción audiovisual.

\section{LA INVESTIGACIÓN REALIZADA}

El estudio realizado, persigue alcanzar los siguientes objetivos:

a) Conocer las universidades públicas y privadas españolas que poseen una televisión universitaria por Internet.

b) Analizar las características fundamentales que presentan dichos programas.

Todo ello en la búsqueda de patrones que puedan conformar ideas para aquellas instituciones, universitarias o no, que deseen poner en funcionamiento una TVIP, 0 que deseen mejorar la existente.

Para ello nos apoyamos en la técnica del análisis de contenido (Bardin, 1986; Krippendorff, 1990), técnica que tiene fuerte tradición en el terreno de la investigación en medios de enseñanza y en tecnología educativa (Barroso y Cabero, 2010).

En su aplicación seguimos las fases tradicionales apuntadas por Bardin (1986) y Krippendorff (1990): preanálisis, formación del sistema categorial, codificación, análisis e interpretación, y elaboración del informe final.

Lo primero que realizamos fue seleccionar nuestro objeto de estudio, que eran las todas las TVIP existentes en las Universidades públicas y privadas, para lo cual nos apoyamos en el listado de universidades existentes en la página web de la CRUE (http://www.crue.org/universidades/Paginas/default.aspx?Mobile=0). Utilizamos por tanto como criterio de selección del universo, el de exhaustividad (Bardin, 1986). 
Posteriormente y para elaborar el sistema categorial de análisis, seguimos la recomendación realizada por Cabero y Loscertales (1998), que señalan que las categorías pueden establecerse por el investigador a partir de diferentes vías: la revisión teórica y conceptual de su objeto de estudio, otros sistemas categoriales previos formulados por otros autores y el éxito obtenido con su construcción, opiniones de expertos y especialistas en el objeto de estudio, y un preacercamiento a los textos de análisis. En nuestro caso, nos apoyamos en tres aspectos: acercamiento a los programas de TVIP de diferentes universidades tanto españolas como extranjeras, revisión teórica de las características y posibilidades que nos ofrece la TVIP, y la consulta a técnicos productores de programas de TVIP.

Indicar que tras realizar una primera versión del sistema categorial, lo validamos por los técnicos responsables de la TVIP del "Secretariado de Recursos Audiovisuales y Nuevas Tecnologías" de la Universidad de Sevilla.

El sistema categorial final elaborado, estaba compuesto por ocho grandes categorías, y diferentes subcategorías. Para su elaboración se tuvieron en cuenta los principios apuntados por Bardin (1986) respecto a las características que deben poseer las buenas categorías: exclusión mutua, homogeneidad, pertinencia, objetividad y fidelidad, y productividad. En la tabla $n-1$, presentamos la versión final del instrumento categorial elaborado.

\begin{tabular}{|c|c|c|}
\hline Categoría & Subcategoría & Descripción \\
\hline \multirow{5}{*}{$\begin{array}{l}\text { Diseño } \\
\text { (Características } \\
\text { físicas del sitio web) }\end{array}$} & Atractivo & Si el sitio web es llamativo y atractivo. \\
\hline & 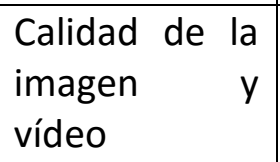 & $\begin{array}{l}\text { Si los videos e imágenes que nos ofrecen } \\
\text { tienen buena calidad. }\end{array}$ \\
\hline & $\begin{array}{l}\text { Visualización } \\
\text { pantalla } \\
\text { completa }\end{array}$ & $\begin{array}{l}\text { Si el sitio web permite, o no, ver los videos } \\
\text { en pantalla completa. }\end{array}$ \\
\hline & $\begin{array}{l}\text { Cortinilla de } \\
\text { vídeo }\end{array}$ & $\begin{array}{l}\text { Existencia de logo institucional al comienzo } \\
\text { de los programas. }\end{array}$ \\
\hline & $\begin{array}{l}\text { Diferentes } \\
\text { idiomas }\end{array}$ & $\begin{array}{l}\text { Posibilidad de cambiar de idioma el sitio } \\
\text { web. }\end{array}$ \\
\hline $\begin{array}{l}\text { Noticias } \\
\text { (Información a la que } \\
\text { se puede acceder en } \\
\text { el sitio web) }\end{array}$ & Actualidad & $\begin{array}{l}\text { Si las noticias que se ofrecen son actuales o } \\
\text { no }\end{array}$ \\
\hline \multirow{2}{*}{$\begin{array}{l}\text { Contenidos de } \\
\text { (Variedad que se } \\
\text { contenidos que en sitio } \\
\text { ofrece en el }\end{array}$} & Videoteca & $\begin{array}{l}\text { Si se encuentran los videos disponibles en } \\
\text { una videoteca }\end{array}$ \\
\hline & Tipos & Si la videoteca está organizada en diversas \\
\hline
\end{tabular}




\begin{tabular}{|c|c|c|}
\hline web). & canales & categorías. \\
\hline \multirow{3}{*}{$\begin{array}{l}\text { Enlaces (Tipos de } \\
\text { contactos que pueda } \\
\text { tener el sitio web } \\
\text { con otras páginas o } \\
\text { redes sociales) }\end{array}$} & Redes sociales & $\begin{array}{l}\text { Si el sitio web dispone o no de redes } \\
\text { sociales. }\end{array}$ \\
\hline & $\begin{array}{l}\text { Enlaces con } \\
\text { otras páginas }\end{array}$ & $\begin{array}{l}\text { Se medirá si el sitio web dispone, o no, de } \\
\text { enlaces con otras páginas web. }\end{array}$ \\
\hline & $\begin{array}{l}\text { Pertenencia a } \\
\text { ATEI }\end{array}$ & $\begin{array}{l}\text { Si se pertenece a ATEI (Televisión Educativa } \\
\text { y Cultural Iberoamericana). }\end{array}$ \\
\hline \multirow{11}{*}{$\begin{array}{l}\text { Servicios (Servicios } \\
\text { ofrecidos en el sitio } \\
\text { web) }\end{array}$} & $\begin{array}{l}\text { Programas } \\
\text { accesibles }\end{array}$ & $\begin{array}{l}\text { Se medirá si se dispone o no de programas } \\
\text { accesibles. }\end{array}$ \\
\hline & $\begin{array}{l}\text { Retransmisión } \\
\text { en directo }\end{array}$ & Si posee retransmisiones en directo. \\
\hline & Contactar & $\begin{array}{l}\text { Posibilidad de ponerte en contacto con la } \\
\text { entidad encargada del sitio web. }\end{array}$ \\
\hline & $\begin{array}{l}\text { Servicio de } \\
\text { Videostreami } \\
\text { ng }\end{array}$ & $\begin{array}{l}\text { Se medirá si se te permite ver los videos a } \\
\text { la carta. Servicio de Videostreaming }\end{array}$ \\
\hline & $\begin{array}{l}\text { Pestaña de } \\
\text { incidencias }\end{array}$ & $\begin{array}{l}\text { Si existe la opción de poder comunicar si } \\
\text { hay algún problema o algún error. }\end{array}$ \\
\hline & $\begin{array}{l}\text { Solicitud de } \\
\text { retransmisión }\end{array}$ & $\begin{array}{l}\text { Posibilidad de solicitar servicios de } \\
\text { ubicación de vídeo o retransmisión de } \\
\text { vídeo. }\end{array}$ \\
\hline & Buscador & Existencia de buscador de contenidos. \\
\hline & $\begin{array}{l}\text { Producción de } \\
\text { contenidos }\end{array}$ & Existencia de producción propia. \\
\hline & $\begin{array}{l}\text { Acceso a } \\
\text { usuarios }\end{array}$ & Acceso a través de un usuario. \\
\hline & $\begin{array}{l}\text { Incrustar } \\
\text { vídeo en } \\
\text { propia web - } \\
\text { compartir }\end{array}$ & $\begin{array}{l}\text { Se ofrece el código html para poder poner } \\
\text { el video en tu propia página web o si se da } \\
\text { la opción de compartirlo en otra red social. }\end{array}$ \\
\hline & Poder & Existe la opción de ofrecer la opinión \\
\hline
\end{tabular}




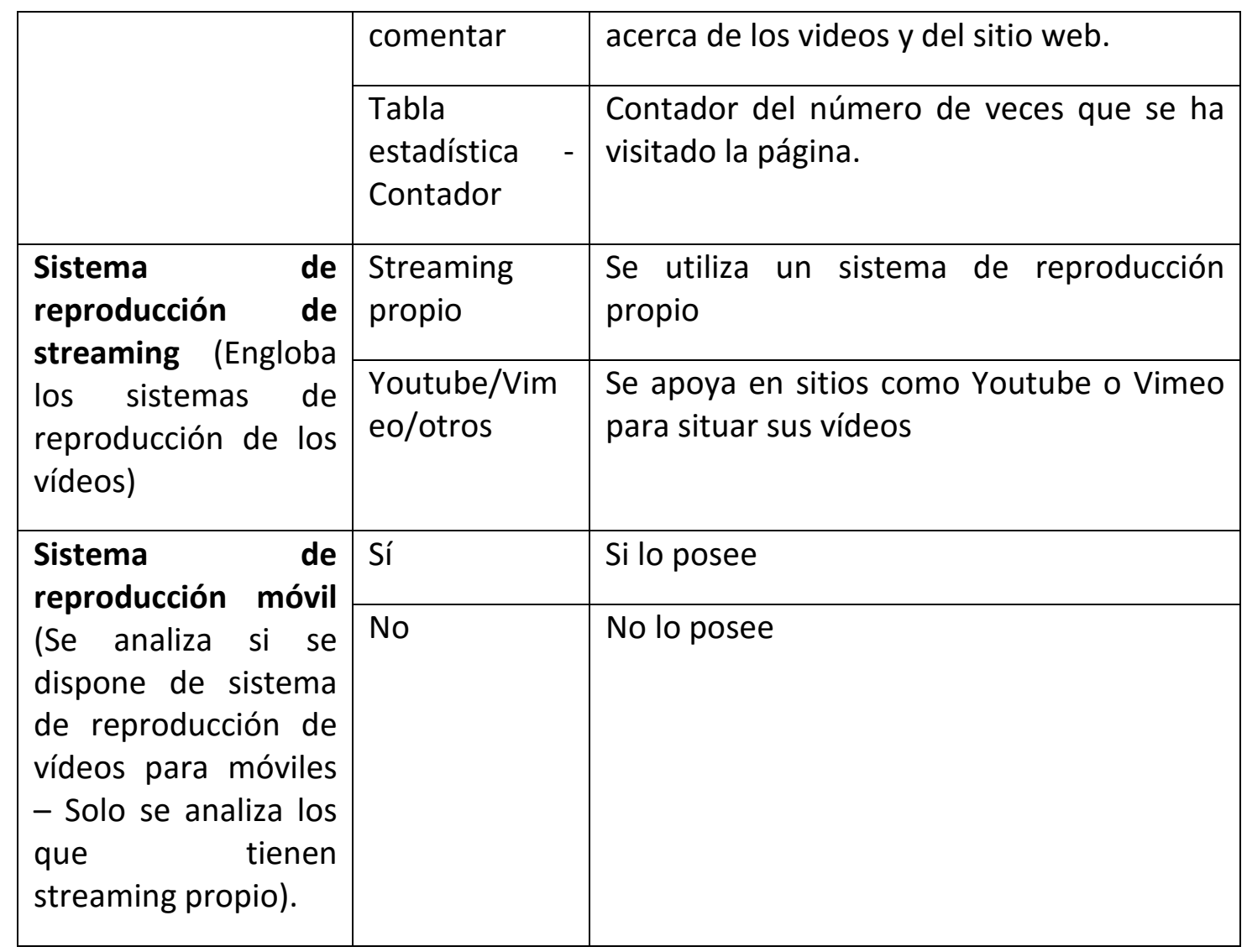

Tabla no 1. Sistema categorial.

Las categorías, por lo general hacían referencia a la existencia o no, del elemento señalado; lo que facilitaba su codificación, y el alcance de acuerdos entre los codificadores. Aunque somos conscientes que la subcategoría "atractivo" es bastante más bien ambigua, insistimos en que para su codificación se apoyaran en el significado que del mismo ofrece la RAE: "que atrae o tiene fuerza para atraer"; de todas formas los resultados en esta categoría deben ser adoptados con cautela.

La codificación fue realizada por dos miembros del equipo de investigación, analizando de forma simultánea el mismo objeto y llegando a acuerdos respecto a la decisión final adoptada. El procedimiento seguido fue: lectura del sistema categorial, administración sobre el objeto a codificar, y codificación definitiva una vez llegado a acuerdo entre los codificadores.

En la tabla no 2 se presentan las universidades analizadas:

\begin{tabular}{|l|l|}
\hline Universidad & Dirección web TVIP \\
\hline Sevilla & http://tv.us.es/ \\
\hline Vigo & tv.uvigo.es/ \\
\hline Murcia & tv.um.es/ \\
\hline Granada & http://cevug.ugr.es/destacados/en-streaming-2.html \\
\hline
\end{tabular}




\begin{tabular}{|c|c|}
\hline Huelva & http://video.uhu.es/1.aspx \\
\hline Castilla la Mancha & http://www.uclmtv.uclm.es/ \\
\hline Politécnica de Valencia & http://www.upv.es/pls/oreg/rtv_web.Ppal?p_idioma=c \\
\hline Santiago de Compostela & http://tv.usc.es/ \\
\hline Salamanca & http://tv.usal.es/ \\
\hline Barcelona & http://www.ub.edu/ubtv/ \\
\hline Burgos & http://www.tvubu.tv/ \\
\hline Carlos III & https://arcamm.uc3m.es/arcamm_3/ \\
\hline Complutense & http://complumedia.ucm.es/ \\
\hline Córdoba & http://aulavirtualtv.uco.es/1.aspx \\
\hline Coruña & www.udctv.es/ \\
\hline Extremadura & http://www.ondacampus.es/tv_index.php?id_aplic=14 \\
\hline Internacional de Andalucía & http://blogs.unia.es/uniatv/ \\
\hline $\begin{array}{l}\text { Internacional Menéndez } \\
\text { Pelayo }\end{array}$ & http://www.uimptv.es/portal.html \\
\hline Jaume I & http://blogs.uji.es/cienciatv/ca/ \\
\hline La Laguna & http://ullmedia.udv.ull.es/ \\
\hline Málaga & http://www.uma.es/ficha.php?id=36079 \\
\hline Miguel Hernández & http://umhtv.umh.es/novedades/ \\
\hline Oviedo & http://mediateca.uniovi.es/inicio \\
\hline País Vasco & http://ehutb.ehu.es/index.html \\
\hline Politécnica de Cartagena & http://www.videoteca.upct.es/index.php \\
\hline Politécnica de Cataluña & http://tv.upc.edu/ \\
\hline Pública de Navarra & https://upnatv.unavarra.es/ \\
\hline Illes Balears & http://dircom.uib.es/es/procediments/pantalles/ \\
\hline Valencia & http://mmedia.uv.es/ \\
\hline Autónoma de Barcelona & http://directe.uab.cat/tv/ \\
\hline
\end{tabular}




\begin{tabular}{|l|l|}
\hline CEU & http://www.ceumedia.es/ \\
\hline Europea de Madrid & http://europeamedia.es/europea-television \\
\hline Europea Miguel de Cervantes & http://www.uemc.es/ \\
\hline Internacional de la Rioja & http://tv.unir.net/ \\
\hline UNED & https://canal.uned.es/ \\
\hline
\end{tabular}

Tabla no 2. TVIP analizadas.

\section{RESULTADOS}

El número de universidades que poseen canales de TVIP en España, son 35 (46,66\%); de las cuales la gran mayoría pertenecen a universidades públicas $(31,62 \%)$ y solamente cuatro a universidades privadas (16\%).

Los resultados que encontramos tras llevar a cabo el análisis de contenido los presentamos en la tabla $n=3$.

\begin{tabular}{|c|c|c|c|c|c|c|}
\hline \multirow[t]{2}{*}{ Subcategoría } & \multicolumn{2}{|l|}{ Públicas } & \multicolumn{2}{|c|}{ Privadas } & \multicolumn{2}{|l|}{ Total } \\
\hline & sí & No & Sí & NO & Sí & NO \\
\hline & $f(\%)$ & $f(\%)$ & $f(\%)$ & $f(\%)$ & $f(\%)$ & $f(\%)$ \\
\hline Atractivo & $\begin{array}{l}18 \\
(58,06 \%)\end{array}$ & $\begin{array}{l}13 \\
(41,93 \%)\end{array}$ & $\begin{array}{l}3 \\
(75 \%)\end{array}$ & $\begin{array}{l}1 \\
(25 \%)\end{array}$ & $21(60 \%)$ & $14(40 \%)$ \\
\hline $\begin{array}{l}\text { Visualización } \\
\text { pantalla completa }\end{array}$ & $\begin{array}{l}28 \\
(100 \%)\end{array}$ & 0 & $\begin{array}{l}4 \\
(100 \%)\end{array}$ & 0 & $\begin{array}{l}32 \\
(100 \%)\end{array}$ & 0 \\
\hline Cortinilla de vídeo & $\begin{array}{l}13 \\
(46,42 \%)\end{array}$ & $\begin{array}{l}15 \\
(53,57 \%)\end{array}$ & $\begin{array}{l}2 \\
(50 \%)\end{array}$ & $\begin{array}{l}2 \\
(50 \%)\end{array}$ & $\begin{array}{l}15 \\
(46.87 \%)\end{array}$ & \begin{tabular}{|l}
17 \\
$(53.12 \%)$
\end{tabular} \\
\hline $\begin{array}{l}\text { Diferentes } \\
\text { idiomas }\end{array}$ & $\begin{array}{l}13 \\
(41,93 \%)\end{array}$ & $\begin{array}{l}18 \\
(58,06 \%)\end{array}$ & 0 & $\begin{array}{l}4 \\
(100 \%)\end{array}$ & $\begin{array}{l}13 \\
(37.14 \%)\end{array}$ & $\begin{array}{l}22 \\
(62.85 \%)\end{array}$ \\
\hline Actualidad & $24(80 \%)$ & $5(20 \%)$ & $\begin{array}{l}4 \\
(100 \%)\end{array}$ & 0 & $\begin{array}{l}28 \\
(84.84 \%)\end{array}$ & \begin{tabular}{|l}
5 \\
$(15.15 \%)$
\end{tabular} \\
\hline Videoteca & $\begin{array}{l}29 \\
(93,54 \%)\end{array}$ & $2(6,45 \%)$ & $\begin{array}{l}3 \\
(75 \%)\end{array}$ & $\begin{array}{l}1 \\
(25 \%)\end{array}$ & $\begin{array}{l}31 \\
(91.17 \%)\end{array}$ & $3(8.82 \%)$ \\
\hline Tipos de canales & $\begin{array}{l}26 \\
(83,87 \%)\end{array}$ & $\begin{array}{l}5 \\
(16,12 \%)\end{array}$ & $\begin{array}{l}3 \\
(75 \%)\end{array}$ & $\begin{array}{l}1 \\
(25 \%)\end{array}$ & $\begin{array}{l}29 \\
(82.87 \%)\end{array}$ & $\begin{array}{l}6 \\
(17.14 \%)\end{array}$ \\
\hline Redes sociales & 21 & 10 & 4 & 0 & 25 & 10 \\
\hline
\end{tabular}




\begin{tabular}{|c|c|c|c|c|c|c|}
\hline & $(67,74 \%)$ & $(32,25 \%)$ & $(100 \%)$ & & $(71.42 \%)$ & $(28.57 \%)$ \\
\hline $\begin{array}{l}\text { Enlaces con otras } \\
\text { páginas }\end{array}$ & $\begin{array}{l}9 \\
(29,03 \%)\end{array}$ & $\begin{array}{l}22 \\
(70,96 \%)\end{array}$ & $\begin{array}{l}1 \\
(25 \%)\end{array}$ & $\begin{array}{l}3 \\
(75 \%)\end{array}$ & $\begin{array}{l}10 \\
(28.57 \%)\end{array}$ & $\begin{array}{l}25 \\
(71.42 \%)\end{array}$ \\
\hline $\begin{array}{l}\text { Pertenencia a } \\
\text { ATEI }\end{array}$ & $3(9,67 \%)$ & $\begin{array}{l}28 \\
(90,32 \%)\end{array}$ & $\begin{array}{l}1 \\
(25 \%)\end{array}$ & $\begin{array}{l}3 \\
(75 \%)\end{array}$ & $4(11.42)$ & $\begin{array}{l}31 \\
(88.57 \%)\end{array}$ \\
\hline $\begin{array}{l}\text { Programas para } \\
\text { discapacitados }\end{array}$ & $2(7,1 \%)$ & $\begin{array}{l}26 \\
(92,8 \%)\end{array}$ & 0 & $\begin{array}{l}4 \\
(100 \%)\end{array}$ & $\begin{array}{l}2 \\
(6.45 \%)\end{array}$ & $\begin{array}{l}29 \\
(93.54 \%)\end{array}$ \\
\hline $\begin{array}{l}\text { Retransmisión en } \\
\text { directo }\end{array}$ & $\begin{array}{l}20 \\
(64,5 \%)\end{array}$ & $\begin{array}{l}11 \\
(35,4 \%)\end{array}$ & 0 & $\begin{array}{l}4 \\
(100 \%)\end{array}$ & $\begin{array}{l}20 \\
(58.82 \%)\end{array}$ & \begin{tabular}{|l}
14 \\
$(41.17 \%)$
\end{tabular} \\
\hline Contactar & $\begin{array}{l}25 \\
(80,6 \%)\end{array}$ & $6(19,3 \%)$ & $\begin{array}{l}4 \\
(100 \%)\end{array}$ & 0 & $\begin{array}{l}29 \\
(82.85 \%)\end{array}$ & $\begin{array}{l}6 \\
(17.14 \%)\end{array}$ \\
\hline $\begin{array}{l}\text { Servicio de } \\
\text { Videostreaming }\end{array}$ & $\begin{array}{l}30 \\
(96,7 \%)\end{array}$ & $1(3,2 \%)$ & $\begin{array}{l}4 \\
(100 \%)\end{array}$ & 0 & $\begin{array}{l}34 \\
(97.14 \%)\end{array}$ & $1(2.85 \%)$ \\
\hline $\begin{array}{ll}\text { Pestaña } & \text { de } \\
\text { incidencias } & \end{array}$ & $2(6,4 \%)$ & $\begin{array}{l}29 \\
(93,5 \%)\end{array}$ & 0 & $\begin{array}{l}4 \\
(100 \%)\end{array}$ & $\begin{array}{l}2 \\
(5.71 \%)\end{array}$ & $\begin{array}{l}33 \\
(94.28 \%)\end{array}$ \\
\hline $\begin{array}{ll}\text { Solicitud } & \text { de } \\
\text { retransmisión } & \end{array}$ & $8(5 \%)$ & $8(5 \%)$ & 0 & $\begin{array}{l}1 \\
(100 \%)\end{array}$ & $\begin{array}{l}8 \\
(47.05 \%)\end{array}$ & $\begin{array}{l}9 \\
(52.94 \%)\end{array}$ \\
\hline Buscador & $\begin{array}{l}29 \\
(93,5 \%)\end{array}$ & $2(6,4 \%)$ & $\begin{array}{l}4 \\
(100 \%)\end{array}$ & 0 & $\begin{array}{l}33 \\
(94.28 \%)\end{array}$ & $\begin{array}{l}2 \\
(5.71 \% \%)\end{array}$ \\
\hline $\begin{array}{l}\text { Producción de } \\
\text { contenidos }\end{array}$ & $\begin{array}{l}10 \\
(83,3 \%)\end{array}$ & $2(16,6 \%)$ & 0 & 0 & $\begin{array}{l}10 \\
(83.33 \%)\end{array}$ & $\begin{array}{l}2 \\
(16.66 \%)\end{array}$ \\
\hline Acceso a usuarios & $\begin{array}{l}11 \\
(35,4 \%)\end{array}$ & $\begin{array}{l}20 \\
(64,5 \%)\end{array}$ & $\begin{array}{l}2 \\
(50 \%)\end{array}$ & $\begin{array}{l}2 \\
(50 \%)\end{array}$ & $\begin{array}{l}13 \\
(37.14 \%)\end{array}$ & $\begin{array}{l}22 \\
(62.85 \%)\end{array}$ \\
\hline $\begin{array}{l}\text { Insertar vídeo en } \\
\text { propia web - } \\
\text { compartir }\end{array}$ & $\begin{array}{l}23 \\
(79,3 \%)\end{array}$ & $6(20,6 \%)$ & $\begin{array}{l}4 \\
(100 \%)\end{array}$ & 0 & $\begin{array}{l}27 \\
(81.81 \%)\end{array}$ & $\begin{array}{l}6 \\
(18.18 \%)\end{array}$ \\
\hline Poder comentar & $5(16,6 \%)$ & $\begin{array}{l}25 \\
(83,3 \%)\end{array}$ & $\begin{array}{l}2 \\
(50 \%)\end{array}$ & $\begin{array}{l}2 \\
(50 \%)\end{array}$ & $\begin{array}{l}7 \\
(20.58 \%)\end{array}$ & \begin{tabular}{|l}
27 \\
$(79.41 \%)$
\end{tabular} \\
\hline $\begin{array}{l}\text { Tabla estadística - } \\
\text { Contador }\end{array}$ & $7(2,58 \%)$ & $\begin{array}{l}24 \\
(77,41 \%)\end{array}$ & 0 & $\begin{array}{l}4 \\
(100 \&)\end{array}$ & $7(20 \%)$ & $28(80 \%)$ \\
\hline Streaming propio & $\begin{array}{l}24 \\
(77,4 \%)\end{array}$ & $7(22,5 \%)$ & $\begin{array}{l}2 \\
(50 \%)\end{array}$ & $\begin{array}{l}2 \\
(50 \%)\end{array}$ & $\begin{array}{l}26 \\
(74.28 \%)\end{array}$ & $\begin{array}{l}9 \\
(25.71 \%)\end{array}$ \\
\hline Youtube/Vimeo/o & $7(22,5 \%)$ & 24 & 2 & 2 & 9 & 26 \\
\hline
\end{tabular}




\begin{tabular}{|l|l|l|l|l|l|l|}
\hline tros & & $(77,4 \%)$ & $(50 \%)$ & $(50 \%)$ & $(25.71 \%)$ & $(74.28 \%)$ \\
\hline $\begin{array}{l}\text { Sistema de } \\
\begin{array}{l}\text { Reproducción } \\
\text { desde sistema } \\
\text { Android }\end{array}\end{array}$ & $\begin{array}{l}17 \\
(70,3 \%)\end{array}$ & $7(29,1 \%)$ & 2 & 0 & 19 & 7 \\
$(100 \%)$ & & $(73.07 \%)$ & $(26.92 \%)$ \\
\hline
\end{tabular}

Tabla $n^{\circ}$ 3. Frecuencias y porcentajes alcanzados.

Antes de comenzar el análisis digamos que no es nuestra pretensión llevar a cabo un análisis comparativo, sino más bien efectuar una visión de conjunto para obtener elementos para la creación de un modelo de TVIP universitaria. El lector interesado puede observar las codificaciones individuales en: http://tecnologiaedu.us.es/tecnoedu/images/stories/anexo.pdf.

Por lo que se refiere a los aspectos del diseño de las TVIP, lo primero a señalar es que fueron codificadas como atractivas en la mayoría de los casos $(f=21,60 \%)$, y que ofrecían en todos los casos la posibilidad de ser observadas a pantalla completa.

No todos los programas que se presentan aparecen con una cortinilla o carátula, donde se ubique la institución que acoge la producción, en concreto no aparece en el $53,12 \%$ ( $f=17)$ de los casos; y ello es independientemente de que la Universidad sea pública o privada (públicas $(f=15,53,57 \%)$ privadas $(f=2,50 \%)$ ). Datos muy similares nos encontramos al analizar el aspecto referido a que la información pueda aparecer en diferentes idiomas; en concreto solamente en el $37,14 \%(f=13)$ de los casos nos lo hemos encontrado, y ninguno en Universidades privadas.

Por lo que se refiere a la actualidad de los programas ofrecidos, y que por tanto las TVIP universitarias no fueran simples repositorios de documentos audiovisuales. $Y$ en este caso tenemos que señalar que la gran mayoría lo alcanza ( $f=31,9117 \%$ ); aspecto que llega al $100 \%$ en las TVIP de las Universidades privadas.

Respecto a los "contenidos", la gran mayoría ofrece una videoteca clasificada con los diferentes programas que han ido produciendo ( $f=31,91,17 \%)$, solamente $3(8,82 \%)$, no lo tenían; videoteca que por lo general suele estar organizada por temáticas y con diferentes canales de emisión ( $\mathrm{f}=29,82,87 \%$ ).

En relación con el uso de redes sociales, indicar que una gran mayoría las incorporaban para el seguimiento y el comentario de los programas ofrecidos ( $f=25,71,42 \%$ ); la no existencia de estas conexiones se dieron todas en las Universidades públicas $(f=10$, $32,25 \%)$. Por lo general no existen enlaces a otras páginas de televisiones universitarias o asociaciones ( $f=25,71,42 \%)$.

Respecto a los servicios que se ofrecen, lo primero a destacar es que en no todas se ofrecía la posibilidad de retransmisiones en directo $(f=20,58,82 \%)$, siendo todas ellas públicas $(f=20,64,5 \%)$. Ello es un indicador de la existencia de servicios de producción audiovisual en las primeras universidades señaladas. Datos muy parecidos nos encontramos al analizar la subcategoría que se refería a la posibilidad de ofrecer un servicio de "contactar" con los responsables del programa, donde en la gran mayoría 
( $\mathrm{f}=29,82,85 \%)$ nos encontrábamos con la posibilidad del citado servicio; destacar que en todas las privadas se ofrecía el mismo. En contraposición a lo anterior, nos hemos encontrado con que en pocas TVIP aparecía un servicio de "incidencias" ( $f=2,5,71 \%$ ); servicio no existente en las privadas.

En pocas TVIP se ofrecía un servicio para que los miembros de la comunidad universitarias, pudieran solicitar la retransmisión de eventos, solamente lo encontramos en $8(47,05 \%)$, y todas ellas eran de Universidades Públicas.

Un servicio casi mayoritario era el del buscador, en concreto el $94,28 \%$ ( $f=33$ ) disponían del mismo; lo que supone la codificación de los programas mediante una serie de metadatos.

Aunque ya hemos realizado algún comentario al respecto de la producción de los contenidos, los datos registrados en la subcategoría "producción de contenidos" que hacía referencia a si la entidad realizaba o no los contenidos que se ofrecían en la TVIP, y reconociendo que en algunos casos ha sido compleja su identificación, sí existe una gran mayoría de entidades que se dedican a ello $(f=10,83,33 \%)$, y todas ellas pertenecían a instituciones universitarias públicas.

El acceso a través de una clave de usuario para determinados servicios, no era un aspecto muy extendido, solamente en 13 (37,14\%) se ofrecía. En las universidades privadas nos encontramos que un $50 \%$ si lo tenían y otro $50 \%$ no disponían de ello.

También era usual que se ofreciera al usuario la posibilidad de poder poner el vídeo en su propia web y compartirlo; en concreto en 27 (81,81\%) se permitía.

Es también de destacar que en pocas TVIP se ofrecía un servicio para poder comentar la producción audiovisual ( $\mathrm{f}=7 ; 20,58 \%$ ); lo cual nos sugiere que las TVIP universitarias han sido más bien concebidas para el visionado y la reproducción de documentos. En la misma dirección nos encontramos con un servicio de contador de visitas y estadísticas, que solo existía en 7 (20\%), y todas ellas de universidades públicas.

Con nuestro análisis quisimos también conocer si existía en las TVIP una zona de documentos audiovisuales accesibles, pensados para personas con algún tipo de discapacidad, y tenemos que señalar que en muy pocas disponían del mismo; solamente dos $(6,45 \%)$ lo ofrecían, y pertenecían a universidades públicas.

Por lo que se refiere a si el sistema de reproducción era específico de la universidad o se apoyaban en un sistema externo de plataforma de vídeo, como Youtube o Vimeo; indicar que la gran mayoría disponía de un servicio propio ( $f=26,74,28 \%$ ). Así como que también la gran mayoría disponía de un servicio que permitía el visionado de los documentos en sistemas Android; en concreto ello era posible en el $73,07 \%(f=19)$. 


\section{CONCLUSIONES}

La primera conclusión que podemos obtener de nuestro trabajo, es que la TVIP es una realidad en las universidades españolas, fundamentalmente en las públicas, pues casi todas, tienen una destinada a la emisión de sus producciones; es decir, se entiende desde las universidades que la TVIP puede ser un recurso, no solo para transmitir contenidos e información académica y científica, sino también para intentar la creación de una marca.

La TVIP que se está desarrollando en las universidades españolas, supera la concepción de ser un simple repositorio de documentos audiovisuales para ser observados mediante videostreaming, y se convierten en canales de emisiones en directo. De todas formas la puesta en acción de esta opción televisiva requiere la existencia de un equipo técnico, destinado a su producción, emisión y diseño.

Lo analizado nos lleva a reclamar la idea de que la existencia de una TVIP, requiere de la puesta en acción de una televisión que supere el simple acontecimiento del modelo emisión audiovisual, y asocie a la misma diferentes servicios para la interacción, la evaluación y el intercambio de información entre los usuarios; es decir, la configuración de modelos que no se rijan por ser simples contenedores o alojadores de vídeos.

Pensamos que también es necesario reclamar un modelo de televisión que no se quede en las posibilidades que ofrece su convergencia con Internet, sino que aproveche los servicios que ofrecen las herramientas de la web 2.0, como los RSS, y las redes sociales; es decir, crear modelos de TVIP que permitan ampliar la participación de los receptores.

Para finalizar nos gustaría llamar la atención respecto a que en las TVIP de las universidades, se le preste especial atención a crear programas específicos para favorecer la inclusión educativa, y que personas con algún tipo de discapacidad puedan acceder también a los mensajes ubicados en ellas.

\section{REFERENCIAS}

AGUADED, I. Y MARÍN, V. (2013). La televisión por Internet: WebTV. En I. Aguaded y J. Cabero, J. (coords.). Tecnologías y medios para la educación en la e-sociedad. (pp. 117-136). Madrid: Alianza.

AGUADED, I. SÁNCHEZ, J. Y MARÍN, V. (2013). Televisión Educativa. Madrid: Síntesis.

ARTOPOULOS, A. (2011). La Sociedad de las cuatro pantallas. Una mirada Latinoamericana. Barcelona: Fundación Telefónica/Ariel.

BARDIN, L. (1986). Análisis de contenido. Madrid: Akal.

BARROSO, J. Y CABERO, J. (2010). La investigación educativa en TIC. Madrid: Síntesis. 
BLUM, R.A. (2013). Television and Screen Writing. London: Routledge.

BRUBAKER, J. (2010). Internet and Television Are Not Substitutes for Seeking Political Information. Communication Research Reports, 27:4, 298-309.

CABERO, J. (2005). ¿Pero sigue existiendo la televisión en un universo global de comunicación? Comunicar, 25, 57-62.

CABERO, J. (2015). Aportes de la televisión universitaria a las industrias culturales y creativas en Iberoamérica. Edmetic, 4, 1, pp. 5-24.

CABERO, J. Y LOSCERTALES, F. (1998). ¿Cómo nos ven los demás? La imagen del profesor y la enseñanza en los medios de comunicación social. Sevilla: SP de la Universidad de Sevilla.

CEBRIÁN HERREROS, M. (2004). Modelos de televisión: generalista, temática y convergente con internet. Barcelona: Paidós.

COOPER, CH. (2007). Television on the Internet: Regulating new ways of viewing. Information \& Communications Technology Law, 16:1, pp. 1-15.

GARCÍA, A., VINADEL, R. Y ALBUIN, N. (2010). Televisión tradicional y televisión móvil. Telos, 83, pp. 84-96.

GONZÁLEZ, P. Y LÓPEZ, N. (2011). La generación digital ante un nuevo modelo de televisión: contenidos y soportes preferidos. Anàlisi, 4, pp. 31-48.

KRIPPENDORFF, K. (1980). Metodología de análisis de contenido. Barcelona: Paidós.

MARTÍNEZ, F. (1998). La televisión educativa iberoamericana. Sevilla: SAV de la Universidad de Sevilla.

OROZCO, G. (2014). TVMorfosis 3. México: Universidad de Guadalajara.

RAMONET, I. (2015). El fin de la televisión de masas. Le monde diplomatique en español, 231, pp. 1-2.

SAID, E. (2009). Transformaciones comunicativas en la era digital. Bogotá: Universidad del Norte.

SCOLARI, C. (2014). Presentación ¿Adónde van las audiencias que no se quedaron? G. Orozco, (coord). TVMorfosis 3. (pp. 7-12). México: Universidad de Guadalajara.

TORRES, G. (2014). El futuro del cine y sus audiencias está en la televisión, particularmente en la TV IP. En G. Orozco, (coord). TVMorfosis 3. (pp. 41-52). México: Universidad de Guadalajara. 
Para citar este artículo:

Cabero, J.; García, L.; Query, P. \& García, D. (2016). La televisión universitaria por Internet: análisis de la situación española. EDUTEC, Revista Electrónica de Tecnología Educativa, 55. Recuperado el dd/mm/aa de http://www.edutec.es/revista 